\title{
ON THE CONSISTENCY OF REYNOLDS STRESS TURBULENCE CLOSURES WITH HYDRODYNAMIC STABILITY THEORY
}

\author{
Charles G. Speziale* \\ Aerospace \& Mechanical Engineering Department \\ Boston University \\ Boston, MA 02215 \\ Ridha Abid \\ High Technology Corporation \\ NASA Langley Research Center \\ Hampton, VA 23681-0001 \\ Gregory A. Blaisdell \\ School of Aeronautics \& Astronautics \\ Purdue University \\ West Lafayette, IN 47907-1282
}

\begin{abstract}
The consistency of second-order closure models with results from hydrodynamic stability theory is analyzed for the simplified case of homogeneous turbulence. In a recent study, Speziale, Gatski and MacGiolla Mhuiris [Phys. Fluids A 2, 1678, 1990] showed that secondorder closures are capable of yielding results that are consistent with hydrodynamic stability theory for the case of homogeneous shear flow in a rotating frame. It is demonstrated in this paper that this success is due to the fact that the stability boundaries for rotating homogeneous shear flow are not dependent on the details of the spatial structure of the disturbances. For those instances where they are - such as in the case of elliptical flows where the instability mechanism is more subtle - the results are not so favorable. The origins and extent of this modeling problem are examined in detail along with a possible resolution based on Rapid Distortion Theory (RDT) and its implications for turbulence modeling.
\end{abstract}

\footnotetext{
${ }^{*}$ This research was supported by the National Aeronautics and Space Administration under NASA Contract No. NAS1-19480 while the first author was in residence at the Institute for Computer Applications in Science and Engineering (ICASE), NASA Langley Research Center, Hampton, VA 23681-0001.
} 


\section{INTRODUCTION}

While Reynolds stress models are usually thought of as being completely empirical and without any theoretical foundation, recent work has demonstrated that this is far too pessimistic an assessment of the current generation of models ${ }^{1-3}$. One encouraging result is the recent discovery that second-order closure models are capable of accurately predicting the stability boundaries for homogeneous shear flow in a rotating frame (see Speziale, Gatski and MacGiolla Mhuiris ${ }^{4}$ ). This is a predictive capability that no eddy viscosity model possesses - including models ranging from the Baldwin-Lomax model ${ }^{5}$ to the standard $K-\varepsilon \operatorname{model}^{6}$. Such better predictions are usually thought to arise from the fact that second-order closures are based on the Reynolds stress transport equation which incorporates more turbulence physics since it is a rigorous consequence of the Navier-Stokes equations. However, it is important to understand to what extent these results genuinely have a sound theoretical basis. This forms the motivation for the present paper.

It will be shown that the reason existing second-order closure models do so well for homogeneous shear flow in a rotating frame is due to the fact that the stability boundaries do not depend on the details of the spatial structure of the disturbances. For homogeneous turbulent flows where this is not the case - such as the example of elliptical flows which exhibit a more subtle instability mechanism involving resonance that only exists over a narrow band of wavenumbers - the results are not so favorable. This problem will be documented in the sections to follow along with a possible resolution. At least some limited consistency with results from hydrodynamic stability theory is needed if Reynolds stress models with greater predictive capabilities are to be obtained. Otherwise, it would not even be possible to predict whether a statistically unsteady turbulence decays or is self-sustaining. The level of consistency that Reynolds stress models should have with results from linear stability theory will be discussed in detail and some illustrative calculations will be presented.

\section{THEORETICAL BACKGROUND}

We will consider incompressible, homogeneous turbulent flows with constant mean velocity gradients, $\partial \bar{v}_{i} / \partial x_{j}$. The Reynolds stress tensor $\tau_{i j}$ is a solution of the transport equation ${ }^{7}$

$$
\dot{\tau}_{i j}=-\tau_{i k} \frac{\partial \bar{v}_{j}}{\partial x_{k}}-\tau_{j k} \frac{\partial \bar{v}_{i}}{\partial x_{k}}+\Pi_{i j}-\varepsilon_{i j}-2 e_{m k j} \Omega_{m} \tau_{i k}-2 e_{m k i} \Omega_{m} \tau_{j k}
$$

for homogeneous turbulence in an arbitrary non-inertial reference frame where $\Omega_{i}$ is the angular velocity of the reference frame and $e_{i j k}$ is the permutation tensor. In (1), $\Pi_{i j}$ and $\varepsilon_{i j}$ are, respectively, the pressure-strain correlation and the dissipation rate tensor given by

$$
\Pi_{i j}=\overline{p\left(\frac{\partial u_{i}}{\partial x_{j}}+\frac{\partial u_{j}}{\partial x_{i}}\right)}
$$




$$
\varepsilon_{i j}=2 \nu \overline{\frac{\partial u_{i}}{\partial x_{k}} \frac{\partial u_{j}}{\partial x_{k}}}
$$

where $u_{i}$ is the fluctuating velocity, $p$ is the fluctuating pressure, $\nu$ is the kinematic viscosity and the overbar represents an ensemble mean $\left(\tau_{i j} \equiv \overline{u_{i} u_{j}}\right.$ is the kinematic Reynolds stress tensor). Second-order closures in turbulence are based on the solution of a modeled version of (1) which arises from a second moment of the Navier-Stokes equations.

In order to achieve closure, models for $\Pi_{i j}$ and $\varepsilon_{i j}$ are needed. In virtually all existing second-order closures, these correlations are modeled in the form

$$
\begin{gathered}
\Pi_{i j}=\varepsilon A_{i j}(\mathbf{b})+K M_{i j k l}(\mathbf{b}) \frac{\partial \bar{v}_{k}}{\partial x_{l}} \\
\varepsilon_{i j}=\frac{2}{3} \varepsilon \delta_{i j}
\end{gathered}
$$

where $K \equiv \frac{1}{2} \tau_{i i}$ is the turbulent kinetic energy, $\varepsilon \equiv \frac{1}{2} \varepsilon_{i i}$ is the (scalar) turbulent dissipation rate, and $b_{i j} \equiv\left(\tau_{i j}-\frac{2}{3} K \delta_{i j}\right) / 2 K$ is the Reynolds stress anisotropy tensor. Eq. (4) is based on a simplified analysis of the Poisson equation for the fluctuating pressure whereas (5) follows from the Kolmogorov assumption of local isotropy ${ }^{7}$. For two-dimensional mean turbulent flows that are homogeneous and near an asymptotic equilibrium state - which includes the cases to be considered in this paper - (4) simplifies to the form (see Speziale, Sarkar and Gatski $\left.^{8}\right)$

$$
\begin{aligned}
& \Pi_{i j}=-2 C_{1} \varepsilon b_{i j}+C_{2} \varepsilon\left(b_{i k} b_{k j}-\frac{1}{3} b_{k l} b_{k l} \delta_{i j}\right)+C_{3} K \bar{S}_{i j} \\
& +C_{4} K\left(b_{i k} \bar{S}_{j k}+b_{j k} \bar{S}_{i k}-\frac{2}{3} b_{k l} \bar{S}_{k l} \delta_{i j}\right)+C_{5} K\left(b_{i k} \bar{W}_{j k}+b_{j k} \bar{W}_{i k}\right)
\end{aligned}
$$

where

$$
\bar{S}_{i j}=\frac{1}{2}\left(\frac{\partial \bar{v}_{i}}{\partial x_{j}}+\frac{\partial \bar{v}_{j}}{\partial x_{i}}\right), \quad \bar{W}_{i j}=\frac{1}{2}\left(\frac{\partial \bar{v}_{i}}{\partial x_{j}}-\frac{\partial \bar{v}_{j}}{\partial x_{i}}\right)+e_{m j i} \Omega_{m}
$$

are the mean rate of strain and absolute mean vorticity tensors and $C_{1}-C_{5}$ are constants.

Virtually all existing second-order closures use a modeled transport equation for the turbulent dissipation rate that is of the general form

$$
\dot{\varepsilon}=-C_{\varepsilon 1} \frac{\varepsilon}{K} \tau_{i j} \frac{\partial \bar{v}_{i}}{\partial x_{j}}-C_{\varepsilon 2} \frac{\varepsilon^{2}}{K}
$$

for homogeneous turbulence. In (8), $C_{\varepsilon 1}$ and $C_{\varepsilon 2}$ are either constants or are functions of the flow invariants which become constants when the turbulence has achieved an asymptotic state. The Speziale, Sarkar and Gatski (SSG) model $^{8}$, the Launder, Reece and Rodi model ${ }^{9}$ 
and the IP model ${ }^{10}$ are all special cases $(6)$ and (8) where $C_{\varepsilon 1}=1.44$ and $C_{\varepsilon 2}$ is 1.83 for the former model and 1.92 for the latter two models.

\section{MODEL PREDICTIONS}

For homogeneous shear flow in a rotating frame (see Figure 1(a))

$$
\frac{\partial \bar{v}_{i}}{\partial x_{j}}=\left(\begin{array}{ccc}
0 & S & 0 \\
0 & 0 & 0 \\
0 & 0 & 0
\end{array}\right), \quad \Omega_{i}=(0,0, \Omega)
$$

where $S$ is the shear rate and $\Omega$ is the angular velocity of the reference frame which are both constants. In homogeneous shear flows, the Reynolds stress anisotropy tensor $b_{i j}$ and the dimensionless shear parameter $S K / \varepsilon$ achieve equilibrium values that are largely independent of the initial conditions (these fixed points are denoted by $\left(b_{i j}\right)_{\infty}$ and $(S K / \varepsilon)_{\infty}$ since they are the asymptotic values obtained in the limit as $t \rightarrow \infty)$. From the contraction of (1), and the results of Speziale and MacGiolla Mhuiris ${ }^{11}$, it follows that for long times

$$
\dot{K}^{*}=\frac{C_{\varepsilon 2}-C_{\varepsilon 1}}{C_{\varepsilon 1}-1}\left(\frac{\varepsilon}{S K}\right)_{\infty} K^{*}
$$

after (8) is made use of (here, $K^{*}=K / K_{0}$ and $\dot{K}^{*}$ is its time derivative with respect to the dimensionless time, $\left.t^{*} \equiv S t\right)$. If $(\varepsilon / S K)_{\infty}>0$, then we have the long time asymptotic solution

$$
K^{*} \sim e^{\lambda t^{*}}
$$

where

$$
\lambda \equiv \frac{C_{\varepsilon 2}-C_{\varepsilon 1}}{C_{\varepsilon 1}-1}\left(\frac{\varepsilon}{S K}\right)_{\infty}
$$

is the dimensionless growth rate. This, of course, corresponds to unstable flow since there is an exponential growth of the turbulent kinetic energy given that $C_{\varepsilon 1}>1$ and $C_{\varepsilon 2}>C_{\varepsilon 1}$.

If $(\varepsilon / S K)_{\infty}=0$, then $\dot{K}^{*} \rightarrow 0$ as $t \rightarrow \infty$ in such a way that the long time asymptotic solution is the power law decay $K^{*} \sim t^{-\beta}$ where the exponent $\beta$ depends on the ratio of the rotation rate to the shear rate, $\Omega / S$, as well as on the model constants in (6) and (8) (see Speziale and MacGiolla Mhuiris $\left.{ }^{11,12}\right)$. The fixed point $(\varepsilon / S K)_{\infty}=0$ exists for all $\Omega / S$, however it is only stable (i.e., a stable focus) outside of the interval

$$
A \leq \frac{\Omega}{S} \leq B
$$

where $A$ and $B$ are determined by the constants in the model. For $A<\Omega / S<B$, a stable fixed point of the focus type, with $(\varepsilon / S K)_{\infty}>0$, exists in parallel with the $(\varepsilon / S K)_{\infty}=0$ 
fixed point that becomes a saddle which is unstable. This exchange of stabilities is illustrated in the bifurcation diagram provided in Figure 2. Virtually all existing second-order closures predict that point $\mathrm{A}$ is approximately at $\Omega / S=-0.09$. Furthermore, second-order closures with $C_{5}=0$ (such as the early proposal of Rotta ${ }^{13}$ ) predict that point $\mathrm{B}$ is somewhat beyond $\Omega / S=0.5$. The SSG model was optimized to perform well for a range of benchmark two-dimensional mean turbulent flows that are near equilibrium; this renders the constant $C_{5}=0.40$ which places point $\mathrm{B}$ at $\Omega / S \approx 0.53$ (see Speziale, Sarkar and Gatski ${ }^{8}$ ). However, it is worth noting that the older Launder, Reece and Rodi ${ }^{9}$ model predicts a premature restabilization at $\Omega / S \approx 0.38$.

It is thus clear that state-of-the-art second-order closures predict that homogeneous shear flow in a rotating frame is unstable for approximately the range

$$
0 \leq \frac{\Omega}{S} \leq 0.5
$$

wherein there is an exponential growth of the turbulent kinetic energy. Discernibly outside of the interval (14) the flow is stable wherein the turbulent kinetic energy undergoes a power law decay (the time evolution of the turbulent kinetic energy in each of these distinct regimes is illustrated in Figure 3 for the SSG model). These results are consistent with linear hydrodynamic stability theory which predicts unstable flow for $0<\Omega / S<0.5$ (see Lezius and Johnston ${ }^{14}$ ). Of course, one expects the nonlinear stability boundaries to be somewhat broader as predicted by the models (it is well known that homogeneous shear flow, where $\Omega / S=0$, is linearly stable but nonlinearly unstable ${ }^{15}$ ). Unfortunately, a detailed comparison with results for the nonlinear stability of rotating homogeneous shear flow is not possible due to the fact that no such studies have apparently been published. Nonetheless, based on previous comparisons with large-eddy simulations ${ }^{8}$, the predictions of second-order closures appear to be very good, at least from a partial quantitative standpoint.

While the stability boundaries of rotating homogeneous shear flow are described surprisingly well by second-order closures, the same is not the case for certain other homogeneous turbulent flows where the instability mechanism is more subtle. An example of such a homogeneous turbulent flow is the case of plane strain with an added solid body rotation in an inertial frame (see Figure 1(b)). This is characterized by the mean velocity gradient tensor

$$
\frac{\partial \bar{v}_{i}}{\partial x_{j}}=\left(\begin{array}{ccc}
0 & \Gamma+\omega & 0 \\
\Gamma-\omega & 0 & 0 \\
0 & 0 & 0
\end{array}\right)
$$

where $\Gamma$ is the strain rate and $\omega$ is the imposed rotation rate which are both constants (here, $\Omega_{i}=0$ since the reference frame is inertial). For $\omega>\Gamma$, we have the case of elliptical flows 
which have been the subject of recent studies in hydrodynamic stability theory (see Bayly ${ }^{16}$ and Landman and Saffman $\left.{ }^{17}\right)$. We have hyperbolic flows for $\omega<\Gamma$.

For this flow, Eqs. (1) and (8) can be written in terms of $b_{i j}, K^{*}$ and $\varepsilon / \Gamma K$ as follows:

$$
\begin{gathered}
\frac{d K^{*}}{d t^{*}}=-\left(4 b_{12}+\frac{\varepsilon}{\Gamma K}\right) K^{*} \\
\frac{d}{d t^{*}}\left(\frac{\varepsilon}{\Gamma K}\right)=4\left(1-C_{\varepsilon 1}\right)\left(\frac{\varepsilon}{\Gamma K}\right) b_{12}+\left(1-C_{\varepsilon 2}\right)\left(\frac{\varepsilon}{\Gamma K}\right)^{2} \\
\frac{d b_{12}}{d t^{*}}=\frac{1}{2} C_{3}-\frac{2}{3}+\left[\frac{1}{2}\left(C_{4}-2\right)-\frac{\omega}{\Gamma}\left(\frac{1}{2} C_{5}-1\right)\right] b_{11}+\left[\frac{1}{2}\left(C_{4}-2\right)\right. \\
\left.+\frac{\omega}{\Gamma}\left(\frac{1}{2} C_{5}-1\right)\right] b_{22}+\left(1-C_{1}-\frac{1}{2} C_{2} b_{33}\right)\left(\frac{\varepsilon}{\Gamma K}\right) b_{12}+4 b_{12}^{2} \\
\frac{d b_{11}}{d t^{*}}=\left[\frac{1}{3}\left(C_{4}-2\right)+\left(C_{5}-2\right)\left(\frac{\omega}{\Gamma}\right)\right] b_{12}+\left[\left(1-C_{1}\right) b_{11}\right. \\
\left.+\frac{1}{2} C_{2}\left(b_{11}^{2}+b_{12}^{2}-\frac{1}{3} I I_{b}\right)\right]\left(\frac{\varepsilon}{\Gamma K}\right)+4 b_{12} b_{11} \\
\frac{d b_{22}}{d t^{*}}=\left[\frac{1}{3}\left(C_{4}-2\right)-\left(C_{5}-2\right)\left(\frac{\omega}{\Gamma}\right)\right] b_{12}+\left[\left(1-C_{1}\right) b_{22}\right. \\
\left.+\frac{1}{2} C_{2}\left(b_{22}^{2}+b_{12}^{2}-\frac{1}{3} I I_{b}\right)\right]\left(\frac{\varepsilon}{\Gamma K}\right)+4 b_{12} b_{22}
\end{gathered}
$$

where $I I_{b} \equiv b_{i j} b_{i j}$ and $t^{*} \equiv \Gamma t$ (here we do not need to write the transport equation for $b_{33}$ since $b_{33}=-b_{11}-b_{22}$ from the traceless property of the anisotropy tensor). Following the same analysis as presented in Speziale and MacGiolla Mhuiris ${ }^{11}$, the fixed points are obtained by setting the derivatives on the left-hand-sides of (17)-(20) to zero and then solving the resulting algebraic equations simultaneously (here, the phase space is four dimensional). Neglecting the quadratic return term for simplicity, since it is small, yields:

$$
\left(b_{12}\right)_{\infty}=-\frac{1}{4} \alpha\left(\frac{\varepsilon}{\Gamma K}\right)_{\infty}
$$

and

$$
\left(\frac{\varepsilon}{\Gamma K}\right)_{\infty}=\frac{1}{C_{1}+\alpha-1}\left[\frac{1}{3}\left(C_{4}-2\right)^{2}-\left(C_{5}-2\right)^{2}\left(\frac{\omega}{\Gamma}\right)^{2}+\frac{4}{\alpha}\left(\frac{2}{3}-\frac{1}{2} C_{3}\right)\left(C_{1}+\alpha-1\right)\right]^{\frac{1}{2}}
$$

for $-\delta<\omega / \Gamma<\delta$. Here, we have 


$$
\delta=\frac{\left[\frac{1}{3}\left(2-C_{4}\right)^{2}+\frac{4}{\alpha}\left(\frac{2}{3}-\frac{1}{2} C_{3}\right)\left(C_{1}+\alpha-1\right)\right]^{\frac{1}{2}}}{2-C_{5}}
$$

given that $\alpha \equiv\left(C_{\varepsilon 2}-1\right) /\left(C_{\varepsilon 1}-1\right) ;(\varepsilon / \Gamma K)_{\infty}=0$ outside of the interval $-\delta<\omega / \Gamma<\delta$. When (21) is substituted into (16) it yields an equation that is identical to (10) except that $(\varepsilon / S K)_{\infty}$ is replaced by $(\varepsilon / \Gamma K)_{\infty}$ which is given by $(22)$ (the expression for $(\varepsilon / S K)_{\infty}$ valid in rotating homogeneous shear flow can be found in Speziale and MacGiolla Mhuiris ${ }^{11}$ for comparison with $(22)$ ).

Analogous to the case of homogeneous shear flow in a rotating frame, existing secondorder closures predict unstable flow for the intermediate band of rotation rate to strain rate ratios, $-\delta<\omega / \Gamma<\delta$, where $(\varepsilon / \Gamma K)_{\infty}>0$ yielding exponential growth (for the SSG model, $\delta \approx 1.34)$. For $\omega / \Gamma$ outside of this interval, $(\varepsilon / \Gamma K)_{\infty}=0$ yielding a power law decay of the turbulent kinetic energy. The fixed point $(\varepsilon / \Gamma K)_{\infty}=0$ exists for all values of $\omega / \Gamma$, but it is only stable outside of the interval $-\delta<\omega / \Gamma<\delta$. This is illustrated by the bifurcation diagram (Figure 4) and the plots of the time evolution of the turbulent kinetic energy (Figure 5) provided for the SSG model. There is the same type of exchange of stabilities (with an identical phase space portrait) as that obtained for the case of rotating homogeneous shear flow. While this stability picture is correct for homogeneous shear flow in a rotating frame, it is decidedly in error for plane strain with an added solid body rotation as given by (15). For $|\omega / \Gamma|>1$ we have the class of elliptical flows which have been shown to be unstable for all finite values of $\omega / \Gamma$ (see Bayly ${ }^{16}$, Landman and Saffman ${ }^{17}$, Pierrehumbert ${ }^{18}$ and Waleffe ${ }^{19}$, the latter of which considers the nonlinear stability). All existing second-order closures erroneously predict a restabilization starting somewhere before $|\omega / \Gamma|=2$. The reason for these poor predictions in contrast to the remarkably good predictions for homogeneous shear flow in a rotating frame will be addressed in the next section.

\section{LINEAR STABILITY ANALYSIS}

A simplified linear stability analysis of the homogeneous flows considered in the previous section will now be presented. Our purpose here is to determine how sensitive the stability boundaries are to the details of the spatial structure of the disturbances. We consider a standard decomposition of the velocity field $v_{i}$ and pressure $p$ of the form

$$
v_{i}=U_{i}+u_{i}^{\prime}, \quad p=P+p^{\prime}
$$

where $U_{i}$ and $P$ represent the velocity and pressure associated with the base flow whereas $u_{i}^{\prime}$ and $p^{\prime}$ represent the disturbance velocity and pressure. The substitution of (24) into the Navier-Stokes equation, in a non-inertial frame, yields the disturbance equation ${ }^{15}$ 


$$
\frac{\partial u_{i}^{\prime}}{\partial t}+U_{k} \frac{\partial u_{i}^{\prime}}{\partial x_{k}}=-u_{k}^{\prime} \frac{\partial U_{i}}{\partial x_{k}}-\frac{\partial p^{\prime}}{\partial x_{i}}+\nu \nabla^{2} u_{i}^{\prime}-2 e_{i j k} \Omega_{j} u_{k}^{\prime}
$$

after the base flow (which also satisfies the Navier-Stokes equation) is subtracted out and terms that are nonlinear in the disturbances, which are assumed small, are neglected. Eq. (25) forms the basis for the linear theory of hydrodynamic stability in non-inertial frames.

The simplified case of spatially uniform disturbances of the form

$$
u_{i}^{\prime}=u_{i}^{\prime}(t), \quad p^{\prime}=p^{\prime}(t)
$$

will be considered. Here, $u_{i}^{\prime}=u_{i}^{\prime}(t)$ implies that $p^{\prime}=p^{\prime}(t)$ after we make use of the incompressibility constraint and the fact that the pressure disturbances must be bounded for the linear stability limit to be valid. After substituting (26) into (25), the simple dynamical system

$$
\dot{u}_{i}^{\prime}+A_{i k} u_{k}^{\prime}=0
$$

is obtained where

$$
A_{i k}=\frac{\partial U_{i}}{\partial x_{k}}+2 e_{i j k} \Omega_{j}
$$

is a constant tensor for the homogeneous flows under consideration. For homogeneous shear flow in a rotating frame, it is clear from (9) that

$$
A_{i k}=\left(\begin{array}{ccc}
0 & S-2 \Omega & 0 \\
2 \Omega & 0 & 0 \\
0 & 0 & 0
\end{array}\right)
$$

Since the non-zero eigenvalues of this matrix are given by

$$
\lambda_{1,2}= \pm \sqrt{2 \Omega(S-2 \Omega)}
$$

it follows that the solution of (27) is of the form

$$
u_{\alpha}^{\prime}=u_{\alpha}^{\prime}(0) e^{-\lambda_{\alpha} t}
$$

relative to the principal axes $x_{\alpha}$. Hence, the kinetic energy $K$ of the disturbances has the asymptotic form

$$
K \propto e^{2 \sqrt{R_{\imath}} t^{*}}
$$

where $t^{*} \equiv S t$ and

$$
R_{i}=2 \frac{\Omega}{S}\left(1-2 \frac{\Omega}{S}\right)
$$


is the rotational Richardson number (see Bradshaw ${ }^{20}$ who defined $R_{i}$ to be the negative of (32)). Hence, with this definition of the Richardson number, the flow is unstable if $R_{i}>0$ or, equivalently, if

$$
0<\frac{\Omega}{S}<\frac{1}{2}
$$

Eq. (33) is identical to the rigorous linear stability result based on a full normal mode analysis (see Lezius and Johnston ${ }^{14}$ ). It is thus clear that here the instability mechanism does not depend on the details of the spatial structure of the disturbances - a result that explains the success of second-order closures which smear out information about the spatial structure of the fluctuations in homogeneous turbulent flows.

For plane strain with an imposed solid body rotation in an inertial frame, the matrix $A_{i k}$ takes the form

$$
A_{i k}=\left(\begin{array}{ccc}
0 & \Gamma+\omega & 0 \\
\Gamma-\omega & 0 & 0 \\
0 & 0 & 0
\end{array}\right)
$$

since, for this case, $A_{i k}=\partial U_{i} / \partial x_{k}$. The non-zero eigenvalues of (34) are as follows:

$$
\lambda_{1,2}= \pm \sqrt{\Gamma^{2}-\omega^{2}}
$$

Thus, implementing the same logic used to arrive at (31), we obtain the asymptotic form

$$
K \propto e^{2 \sqrt{1-\omega^{2} / \Gamma^{2}} t^{*}}
$$

where $t^{*} \equiv \Gamma t$. Hence, we have unstable flow for $|\omega / \Gamma|<1$ : a result that is comparable to the bound of $|\omega / \Gamma|<1.34$ obtained from the SSG second-order closure. However, unlike the corresponding result obtained for rotating homogeneous shear flow, this stability result is decidedly not correct. Recent linear stability studies based on a full normal mode analysis - conducted by Bayly ${ }^{16}$ for the inviscid case and Landman and Saffman ${ }^{17}$ for the viscous case - have indicated that there is unstable flow for all finite values of $\omega / \Gamma$ in the elliptical flow domain where $|\omega / \Gamma|>1$. Furthermore, it should be noted that this flow is nonlinearly unstable for all finite values of $\omega / \Gamma$ (see Waleffe $\left.{ }^{19}\right)$. This is a somewhat surprising result in that strong rotation is often thought of as a stabilizing effect. However, for rotation dominated elliptical flows, the instability mechanism is based on a more subtle resonance effect that only exists over a narrow band of wavenumbers ${ }^{16}$. This feature causes considerable difficulties in conducting direct numerical simulations of elliptical flows (see Blaisdell and Shariff $\left.{ }^{21}\right)$.

Interestingly enough, second-order closures predict the same topological structure of the instability for plane strain in a rotating frame of reference as that manifested in the bifurcation diagram shown in Figure 3 for the hyperbolic-elliptical flow case. However, unlike for 
the latter case, this instability picture is correct for plane strain in a rotating frame which bears some similarities to rotating shear flow ${ }^{22}$. For plane strain in a rotating frame,

$$
\frac{\partial U_{i}}{\partial x_{j}}=\left(\begin{array}{ccc}
0 & \Gamma & 0 \\
\Gamma & 0 & 0 \\
0 & 0 & 0
\end{array}\right), \quad \Omega_{i}=(0,0, \Omega)
$$

rendering

$$
A_{i k}=\left(\begin{array}{ccc}
0 & \Gamma-2 \Omega & 0 \\
\Gamma+2 \Omega & 0 & 0 \\
0 & 0 & 0
\end{array}\right) .
$$

The non-zero eigenvalues of (37) are given by

$$
\lambda_{1,2}= \pm \sqrt{\Gamma^{2}-4 \Omega^{2}}
$$

which yields a real positive eigenvalue - and, hence, unstable flow - if $|\Omega / \Gamma|<\frac{1}{2}$. This is in line with RDT results which predict a restabilization for values of $|\Omega / \Gamma|$ that exceed 0.7 or even slightly smaller values (F. Godeferd, private communication). The fixed point analysis for the SSG second-order closure in this case is qualitatively the same as that for plane strain with an added solid body rotation shown earlier. It yields unstable flow for

$$
-0.6<\frac{\Omega}{\Gamma}<0.6
$$

which is in line with RDT and with the results of the simplified linear stability analysis presented above. The time evolution of the RDT solution for the turbulent kinetic energy is displayed in Figure 6 for $\Omega / \Gamma=0.3$ and $\Omega / \Gamma=2$ which, respectively, are in the unstable and stable regime (F. Godeferd, private communication). From a qualitative standpoint, these results show a remarkable similarity to the results obtained from second-order closures shown in Figure 5 for the companion problem of plane strain with an added solid body rotation. Second-order closures do not see the qualitative difference between plane strain in a rotating frame and plane strain with an added solid body rotation.

Finally, we would like to discuss the role that Rapid Distortion Theory (RDT) can play in the development of Reynolds stress turbulence closures that are more consistent with hydrodynamic stability theory results for homogeneous flows. We first note that a general RDT analysis is equivalent to a linear stability analysis for homogeneous flows - a fact that is not often stated explicitly and, therefore, not widely appreciated outside of the field of theoretical turbulence. For homogeneous turbulence,

$$
\frac{\partial \tau_{i j}}{\partial x_{j}}=0
$$


and, thus, the evolution equation for the fluctuating velocity reduces to

$$
\frac{\partial u_{i}}{\partial t}+\bar{v}_{k} \frac{\partial u_{i}}{\partial x_{k}}=u_{k} \frac{\partial u_{i}}{\partial x_{k}}-u_{k} \frac{\partial \bar{v}_{i}}{\partial x_{k}}-\frac{\partial p}{\partial x_{i}}+\nu \nabla^{2} u_{i}-2 e_{i j k} \Omega_{j} u_{k}
$$

in an arbitrary non-inertial frame. RDT deals with solutions to the linearized version of (39), where the nonlinear convective term $u_{k} \partial u_{i} / \partial x_{k}$ is neglected ${ }^{23}$. As a result of these considerations, the Reynolds-averaged Navier-Stokes equation is identical to the NavierStokes equation itself eliminating any distinction between $\bar{v}_{k}$ and $U_{k}$. Under these conditions, (39) becomes identical to (25), which renders an RDT analysis equivalent to a linear stability analysis.

It is thus clear that improved consistency with linear stability theory results would be achieved if Reynolds stress turbulence closures were more consistent with RDT. In homogeneous turbulence, the RDT solution has been demonstrated to constitute an excellent approximation to the full Navier-Stokes equations, for at least short elapsed times, in rapidly distorted flows where

$$
\left\|\frac{\partial \bar{v}_{i}}{\partial x_{j}}\right\|>>\frac{\varepsilon_{0}}{K_{0}}
$$

given that $\|\cdot\|$ denotes any suitable norm. Unfortunately, within this domain where it is expected to be an excellent approximation, existing second-order closures have been shown to perform poorly compared to RDT. This has motivated Reynolds ${ }^{24}$ and Kassinos and Reynolds ${ }^{25}$ to attempt to develop Reynolds stress closures that are fully consistent with RDT by incorporating the structure tensor that includes information on the dimensionality of the turbulence. While this recent work of Reynolds and co-workers has a worthwhile goal - and contains many interesting new ideas - questions can be raised about whether or not it is possible to develop any one-point closure that is consistent with RDT for all flows. Two-point closures, which contain full spatial information, are probably needed for this purpose ${ }^{26}$.

As stated earlier, it has been clearly demonstrated during the past decade that existing second-order closures perform poorly in the RDT limit. However, it must be noted that while the development of second-order closures that are consistent with RDT for the early transients of rapidly distorted turbulent flows would help, it is not sufficient to guarantee consistency with linear hydrodynamic stability theory. It is possible to construct a secondorder closure that in the RDT limit has approximately the correct behavior but for weaker distortions behaves erroneously. Thus, the first author has recently pursued an approach that seeks to establish consistency with RDT only for a limited set of benchmark results, without compromising the performance of second-order closures in more weakly distorted turbulent flows where nonlinear effects play a major role ${ }^{27}$. 


\section{CONCLUSION}

An analysis of the consistency of existing second-order closure models with hydrodynamic stability theory results has been conducted for two basic homogeneous turbulent flows. Most notably in this regard, it is crucial for consistency to ensure that a turbulence model does not erroneously predict a decaying turbulent kinetic energy in a flow that is linearly unstable. In contrast to the earlier, and highly encouraging, results obtained for homogeneous shear flow in a rotating frame, it was found that the predictions are poor for the case of elliptical flows. The existing hierarchy of second-order closures predict that elliptical flows restabilize when the rotation rate exceeds the strain rate by any discernible amount whereas recent linear stability analyses indicate definitively that the flow is unstable for all finite rotation rates. It was found that this difficulty results from the fact that the instability mechanism is more subtle in elliptical flows. It encapsulates a resonance effect that exists only over a narrow band of wavenumbers which yields sensitivity to the spatial structure of the disturbances - information that is smeared out in homogeneous turbulence when Reynolds averaging is implemented. On the other hand, the stability boundaries for homogeneous shear flow and plane strain in a rotating frame are relatively insensitive to the spatial structure of the disturbances since they can be obtained from a stability analysis based on spatially uniform disturbances.

It is argued that better consistency with the results of linear stability theory would be achieved if models were more consistent with RDT. However, full consistency with linear stability theory would require a model that is consistent with RDT but within a framework that yields growth rates for any unstable flow that are bounded below by the rapid distortion limit (milder distortions where nonlinear interactions play an important role should, in general, be less stable than the linear limit of RDT). As mentioned earlier, despite the very interesting work of Reynolds and co-workers ${ }^{24,25}$, it is debatable as to whether any one-point closure can be developed that is fully consistent with RDT for all flows. Nonetheless, it does appear to be useful to have at least some limited consistency with RDT in certain benchmark homogeneous turbulent flows (Speziale and $\mathrm{Xu}^{27}$ have attempted a simpler approach wherein a relaxation time and non-equilibrium Padé approximation is made around the equilibrium solution of the traditional hierarchy of second-order closures). This more limited approach may be more practical considering the fact that the inconsistencies are not that widespread. Homogeneous turbulent flows involving strains or shear in a rotating frame - as well as hyperbolic flows - are well described. A detailed comparison of a variety of second-order closures with DNS data bases for elliptical flows, hyperbolic flows and plane strain in a rotating frame will be the subject of a future paper. 


\section{ACKNOWLEDGEMENTS}

The first author (CGS) also acknowledges the support of the Office of Naval Research under Grant No. N00014-94-1-0088 (ARI on Nonequilibrium Turbulence, Dr. L.P. Purtell, Program Officer). RA acknowledges the support of NASA Langley Research Center under Contract No. NAS1-20059. GAB acknowledges support from the NASA/ASEE Summer Faculty Fellowship Program. The authors are indebted to F. Godeferd for providing us with his RDT calculations of plane strain in a rotating frame.

\section{References}

[1] C.G. Speziale, "Analytical Methods for the Development of Reynolds Stress Closures in Turbulence," Ann. Rev. Fluid Mech. 23, 107 (1991).

[2] V. Yakhot and S.A. Orszag, "Renormalization Group Analysis of Turbulence I. Basic Theory," J. Sci. Comp. 1, 3 (1986).

[3] C.G. Speziale, "Modeling of Turbulent Transport Equations," in Simulation and Modeling of Turbulent Flows, (T.B. Gatski, et al., eds.) Oxford University Press, to appear.

[4] C.G. Speziale, T.B. Gatski and N. MacGiolla Mhuiris, "A Critical Comparison of Turbulence Models for Homogeneous Shear Flows in a Rotating Frame," Phys. Fluids A 2, $1678(1990)$.

[5] B.S. Baldwin and H. Lomax, "Thin Layer Approximation and Algebraic Model for Separated Turbulent Flows," AIA A Paper No. 78-257 (1978).

[6] B.E. Launder and D.B. Spalding, "The Numerical Computation of Turbulent Flows," Comput. Methods Appl. Mech. Eng. 3, 269 (1974).

[7] J.O. Hinze, Turbulence (McGraw-Hill, New York, 1975).

[8] C.G. Speziale, S. Sarkar and T.B. Gatski, "Modeling the Pressure-Strain Correlation of Turbulence: An Invariant Dynamical Systems Approach," J. Fluid Mech. 227, 245 (1991).

[9] B.E. Launder, G. Reece and W. Rodi, "Progress in the Development of a Reynolds Stress Turbulence Closure," J. Fluid Mech. 68, 537 (1975).

[10] B.E. Launder, "Phenomenological Modeling: Present and Future," Int. J. Heat 83 Fluid Flow 10, 282 (1989). 
[11] C.G. Speziale and N. MacGiolla Mhuiris, "On the Prediction of Equilibrium States in Homogeneous Turbulence," J. Fluid Mech. 209, 591 (1989).

[12] C.G. Speziale and N. MacGiolla Mhuiris, "Scaling Laws for Homogeneous Turbulent Shear Flows in a Rotating Frame," Phys. Fluids A 1, 294 (1989).

[13] J.C. Rotta, "Recent Attempts to Develop a Generally Applicable Calculation Method for Turbulent Shear Flow Layers," AGARD CP-93 (1972).

[14] D.K. Lezius and J.P. Johnston, "Roll-Cell Instabilities in Rotating Laminar and Turbulent Channel Flow," J. Fluid Mech. 77, 153 (1976).

[15] P.G. Drazin and W.H. Reid, Hydrodynamic Stability, (Cambridge University Press, New York, 1981).

[16] B.J. Bayly, “Three-Dimensional Instability of Elliptical Flow," Phys. Rev. Letters 57, $2160(1986)$.

[17] M.J. Landman and P.G. Saffman, "The Three-Dimensional Instability of Strained Vortices in a Viscous Fluid," Phys. Fluids 30, 2339 (1987).

[18] R.T. Pierrehumbert, "Universal Short-Wave Instability of Two-Dimensional Eddies in an Inviscid Fluid," Phys. Rev. Letters 57, 2157 (1986).

[19] F. Waleffe, "The Three-Dimensional Instability of Strained Vortices," Phys. Fluids A 2, $76(1990)$.

[20] P. Bradshaw, "The Analogy Between Streamline Curvature and Bouyancy in Turbulent Shear Flow," J. Fluid Mech. 36, 177 (1969).

[21] G.A. Blaisdell and K. Shariff, "Homogeneous Turbulence Subjected to Mean Flow with Elliptic Streamlines," in Studying Turbulence Using Numerical Simulation Databases V, (P. Moin, et al., eds.), p. 355, Stanford University Press, 1994.

[22] C. Cambon, J.P. Benoit, L. Shao and L. Jacquin, "Stability Analysis and Large-Eddy Simulation of Rotating Turbulence with Organized Eddies," J. Fluid Mech. 278, 175 (1994).

[23] W.C. Reynolds, "Fundamentals of Turbulence for Turbulence Modeling and Simulation," in Lecture Notes for Von Karmań Institute, AGARD Lecture Series No. 86, p. 1, NATO, New York, 1987. 
[24] W.C. Reynolds, "Towards a Structure-Based Turbulence Model," in Studies in Turbulence (T.B. Gatski, et al., eds.), p. 76, Springer, New York, 1991.

[25] S.C. Kassinos and W.C. Reynolds, "A Structure-Based Model for the Rapid Distortion of Homogeneous Turbulence," Stanford University Technical Report TF-61 (1994).

[26] T.T. Clark and C. Zemach, "A Spectral Model Applied to Homogeneous Turbulence," Phys. Fluids 7, 1674 (1995).

[27] C.G. Speziale and X.H. Xu, "Towards the Development of Second-Order Closure Models for Non-Equilibrium Turbulent Flows," Phys. Fluids, submitted for publication. 


\section{LIST OF FIGURES}

Figure 1. Schematic of the flows considered. (a) Homogeneous shear flow in a rotating frame, and (b) Plane strain with an added solid body rotation.

Figure 2. Bifurcation diagram for second-order closures corresponding to homogeneous shear flow in a rotating frame.

Figure 3. Time evolution of the turbulent kinetic energy for homogeneous shear flow in a rotating frame: predictions of the SSG second-order closure model for an initial condition of $S K_{0} / \varepsilon_{0}=4$.

Figure 4. Bifurcation diagram of the SSG second-order closure model corresponding to plane strain turbulence with an added solid body rotation (elliptical flows correspond to $|\omega / \Gamma|>1)$.

Figure 5. Time evolution of the turbulent kinetic energy for plane strain turbulence with an added solid body rotation: predictions of the SSG second-order closure model for an initial condition of $\Gamma K_{o} / \varepsilon_{0}=2$.

Figure 6. Time evolution of the turbulent kinetic energy for plane strain in a rotating frame: RDT calculations of F. Godeferd. 